\title{
Femtosecond THz Studies of Intra-Excitonic Transitions
}

\author{
Rupert Huber, Ben A. Schmid, Robert A. Kaindl, and Daniel S. Chemla \\ Department of Physics, University of California at Berkeley and Materials Sciences Division, E. \\ O. Lawrence Berkeley National Laboratory, 1 Cyclotron Road, Berkeley, CA 94720, USA
}

\begin{abstract}
:
Few-cycle $\mathrm{THz}$ pulses are employed to resonantly access the internal fine structure of photogenerated excitons in semiconductors, on the femtosecond time scale. This technique allows us to gain novel insight into many-body effects of excitons and reveal key quantum optical processes. We discuss experiments that monitor the densitydependent renormalization of the binding energy of a high-density exciton gas in GaAs/ $/ \mathrm{Al}_{0.3} \mathrm{Ga}_{0.7} \mathrm{As}$ quantum wells close to the Mott transition. In a dilute ensemble of $3 \mathrm{p}$ excitons in $\mathrm{Cu}_{2} \mathrm{O}$, stimulated $\mathrm{THz}$ emission from internal transitions to the energetically lower $2 \mathrm{~s}$ state is observed at a photon energy of $6.6 \mathrm{meV}$, with a cross section of $10^{-14}$ $\mathrm{cm}^{2}$. Simultaneous interband excitation of both exciton levels drives quantum beats, which cause efficient $\mathrm{THz}$ emission at the difference frequency. By extending this principle to various other exciton resonances, we develop a novel way of mapping the fine structure by two-dimensional $\mathrm{THz}$ emission spectroscopy.
\end{abstract}




\section{Introduction}

In the dilute limit, excitons in semiconductors may be regarded as Coulomb-bound quasiparticles of a conduction electron with a defect electron (hole) [1,2]. Starting with the quantized internal fine structure, there is a wealth of compelling analogies to atoms. This has motivated research aimed at using excitons as tailorable model systems for atomic gases, potentially implying the perspective of novel quantum collective states of excitons [3].

Nevertheless, there are limits to the atomic analogy. Electron-hole pairs are inherently unstable due to ultrafast recombination. Due to efficient dielectric screening and low effective masses of charge carriers in semiconductors, excitons feature typical binding energies in the meV range, orders of magnitude lower than the atomic Rydberg constant. As complex many-body states embedded in a crystal lattice, excitons strongly interact with lattice vibrations, other excitons, as well as charge carriers. Depending on excitation density and temperature, intriguing new phases may occur. Such phenomena range from molecule-like complexes such as biexcitons via the exciton Mott transition [4,5], to the highly controversially discussed Bose Einstein condensation (BEC) [6-8].

Up to now, most experiments that are targeted to unravel the interplay of these phenomena have studied interband resonances in the visible and infrared spectral range. Since these techniques monitor the creation or annihilation of electron-hole pairs, they are only indirectly sensitive to existing exciton populations [9]. Moreover, they are subject to additional selection rules and often detect only a subset of excitons with vanishing center-of-mass momenta. In particular, promising systems for potential BEC of 
excitons couple only weakly to radiative interband transitions. A lively discussion has been centered on the interpretation of optical spectroscopy [5,8-11].

In contrast, femtosecond terahertz $(\mathrm{THz})$ technology $(1 \mathrm{THz} \approx 4.1 \mathrm{meV})$ has been advanced in recent years to directly trace low-energy excitations in non-equilibrium electron-hole systems [12-18]. Via few-cycle THz pulses one may couple resonantly to internal exciton transitions $[12,15,17,18]$ to explore novel quantum optical processes and shed new light on the complex phase diagram of electron-hole excitations in semiconductors.

In the following section, we briefly review the state of the art of broadband and ultrabroadband $\mathrm{THz}$ technology. These tools are then exploited to study a twodimensional exciton gas at high densities close to the Mott criterion [17], as discussed in section 3. The $\mathrm{THz}$ data provide direct insight into the many-body renormalization of the exciton fine structure. In section 4, a novel analogy between atoms and excitons is discussed: the first observation of stimulated emission of electromagnetic radiation from intra-excitonic transitions [18]. Section 5 reports a new approach to intra-excitonic spectroscopy based on $\mathrm{THz}$ generation by inter-level quantum beats.

\section{Optical pump - THz probe experiments}

The experimental scheme employed in this work is optical pump - $\mathrm{THz}$ probe spectroscopy: A first spectrally shaped femtosecond pulse in the visible or near-infrared domain generates excitons or unbound electron-hole pairs via an interband absorption process. A subsequent ultrashort THz pulse probes the internal response of the bound $e-h$ pairs with femtosecond temporal resolution. 
In recent years, a variety of techniques for generating ultrashort $\mathrm{THz}$ transients has been developed [20]. We employ optical rectification of intense near-infrared laser pulses in nonlinear optical crystals as a particularly versatile approach: Difference frequency generation within the broad spectrum of a single femtosecond light pulse yields inherently phase-locked electromagnetic waves. While the idea was initially exploited for far-infrared transients with frequencies typically up to a few $\mathrm{THz}$, recent years have seen a rapid extension towards the near-infrared domain. Ever shorter pump pulses, efficient and tuneable phase-matching schemes [21,22], and a variety of crystal materials with high $\chi^{(2)}$ nonlinearities, such as $\mathrm{ZnTe}, \mathrm{GaP}, \mathrm{GaSe}$, or $\mathrm{LiIO}_{3}$, have enabled this vast progress [20-24]. Fig. 1(d) displays selected $\mathrm{THz}$ spectra covering the far- and midinfrared, as obtained by a variety of pump spectra and emitter crystals described in the figure caption. Phase matched optical rectification of $7 \mathrm{fs}$ visible light pulses in a thin birefringent $\mathrm{LiIO}_{3}$ emitter very recently enabled multi-THz spectra spanning beyond an optical octave, with center frequencies of up to $100 \mathrm{THz}$ (photon energy $>400 \mathrm{meV}$ ) [curve (iv) of Fig. 1(d)] [24]. New perspectives open up by the possibilities to generate $\mathrm{THz}$ pulses with $\mathrm{MV} / \mathrm{cm}$ peak electric fields in a broad range and precisely shape the wave form of multi-THz field transients $[25,26]$.

Much of the uniqueness of $\mathrm{THz}$ spectroscopy arises from the availability of amplitude- and phase-sensitive detectors, recording the real-time evolution of the oscillating electric field of the infrared pulses [Fig. 1(a)-(c)]. Free-space electro-optic sensing [27] has now been advanced to measure electromagnetic field transients with frequency components ranging from $100 \mathrm{GHz}$ to beyond $100 \mathrm{THz}[28,22,23]$ (see Fig. 1). The spectral window accessible with phase-locked generation and field-resolved 
detection of $\mathrm{THz}$ pulses thus now covers exciton binding energies of virtually all relevant semiconductors.

In the pump-probe experiment, a well-defined $\mathrm{THz}$ field transient is propagated through the semiconductor sample. By electro-optic detection, we characterize both the reference field $E_{\mathrm{THz}}$ transmitted through the sample in equilibrium (no excitons present) and its pump-induced change $\Delta E_{\mathrm{THz}}$ after a fixed pump-probe delay time $\Delta t$, with amplitude and phase. From this information, the entire complex-valued optical conductivity $\sigma(\omega)$ and its transient change $\Delta \sigma(\omega, \Delta t)$ are obtained as a function of the delay time $\Delta t[29,14-18]$. The retrieval algorithm accounts for the precise sample geometry via a transfer matrix formalism [19]. The full $\mathrm{THz}$ dielectric response may be written as $\sigma(\omega)=\sigma_{1}(\omega)-i \omega \varepsilon_{0}\left(1-\varepsilon_{1}(\omega)\right)$. The real part of the optical conductivity $\sigma_{1}(\omega)$ is a measure of the absorbed power density, while the real part of the dielectric function $\varepsilon_{1}(\omega)$ provides a measure of the out-of-phase, inductive response. In the following sections, we will discuss optical pump $-\mathrm{THz}$ probe experiments that reveal novel analogies of excitons and atoms as well as important limitations of this picture.

\section{THz study of a high-density exciton gas in GaAs quantum wells}

A particularly intriguing region of the phase diagram of electron-hole systems in semiconductors is given by the high-density limit of exciton gases close to the so-called Mott transition $[1,2,4,5]$ : Charge-neutral excitons, whose ground state excitation is the transition from $1 \mathrm{~s}$ to $2 \mathrm{p}$ levels, form at sufficiently low densities and temperatures. With increasing densities these bound states are distinctly modified due to many-body effects, 
ultimately depleting the population of bound states. The system then undergoes a crossover into a conductive phase with a continuous low-energy excitation spectrum. In contrast to indirect interband probes, $\mathrm{THz}$ pulses resonantly monitor the characteristic low-energy spectra, providing an immediate measure of binding energies, as the system approaches the Mott transition.

Quasi-2D excitons in quantum wells are particularly well-suited for these investigations, owing to enhanced excitonic binding and sharp optical resonances [30]. Our sample consists of a stack of ten undoped 14-nm-wide GaAs wells separated by 10nm-wide $\mathrm{Al}_{0.3} \mathrm{Ga}_{0.7} \mathrm{As}$ barriers. The near infrared absorption spectrum [Fig. 2(a)] is dominated by the $1 \mathrm{~s}$ heavy-hole $(1 \mathrm{sHH})$ exciton line at $1.540 \mathrm{eV}$. Its linewidth of 0.8 meV (FWHM) attests to a high sample quality. With increasing energy the 2 s heavy-hole, the $1 \mathrm{~s}$ light-hole exciton, and the band-to-band continuum follow. The near-infrared excitation spectrum [dotted curve, Fig. 1(a)] is tuned to resonantly generate 1s excitons.

Fig. 2 displays photo-induced THz conductivity spectra $\Delta \sigma_{1}(\omega)$ and dielectric function changes $\Delta \varepsilon_{1}(\omega)$ at a delay time $\Delta t=2.5 \mathrm{ps}$ after near-infrared excitation. For the lowest density of $n=2 \times 10^{10} \mathrm{~cm}^{-2}$, a narrow asymmetric peak in $\Delta \sigma_{1}(\omega)$ at $\hbar \omega=7 \mathrm{meV}$ witnesses the existence of bound $e$ - $h$ pairs. The maximum arises from the transition from $1 \mathrm{~s}$ to $2 \mathrm{p}$ quantum states, while the high-energy shoulder corresponds to transitions from 1s into higher bound and continuum states. The conductivity vanishes at low frequencies, confirming the insulating nature of the dilute gas. The dispersive zero crossing of $\Delta \varepsilon_{1}$ at $\hbar \omega=7 \mathrm{meV}$ is characteristic of the intra-excitonic oscillator. 
With increasing pump density, profound changes occur in the THz response of Fig. 2: (i) The area enclosed by the conductivity curve $\Delta \sigma_{1}(\omega)$, i.e. the oscillator strength in the $\mathrm{THz}$ window, increases. (ii) The observed $\mathrm{THz}$ resonance strongly broadens. (iii) Both the spectral maximum of $\Delta \sigma_{1}(\omega)$ and the zero crossing of $\Delta \varepsilon_{1}(\omega)$ shift to lower frequencies. At the highest experimentally demonstrated density, the conductivity rises monotonically towards lower frequencies and a zero crossing of the dielectric function is not detected. This behavior indicates a conductive phase in the absence of excitonic resonances.

For a first-order analysis, we model the experimentally determined $\mathrm{THz}$ response (Fig. 2) via the straightforward dielectric function of two-dimensional excitons

$$
\varepsilon_{X}(\omega)=\varepsilon_{\infty}+\frac{n_{X} e^{2}}{\varepsilon_{0} m} \sum_{q} \frac{f_{1 s, q}}{\left(\omega_{1 s, q}^{2}-\omega^{2}\right)-i \omega \Gamma_{X}}
$$

where $\varepsilon_{\infty}$ and $\varepsilon_{0}$ are the background and vacuum dielectric constants, respectively, and $e$ and $m$ are elementary charge and the reduced exciton mass. The oscillator strengths for internal transitions from 1s to higher bound and unbound states are calculated from twodimensional exciton wave functions $[2,31]$. We vary the exciton density $n_{\mathrm{X}}$, the energy position $E_{1 \mathrm{~s}-2 \mathrm{p}}=\hbar \omega_{1 \mathrm{~s}, 2 \mathrm{p}}$, and the phenomenological exciton broadening parameter $\Gamma_{\mathrm{X}}$ to fit the measured conductivity curves. While agreement is good for low densities, at elevated pump powers Eq. (1) does not reproduce both conductivity and dielectric function changes simultaneously (cf. broken curve in Fig. 2). This problem may be resolved by considering additional low-frequency spectral weight in the form of a coexisting plasma 
of unbound $e$ - $h$ pairs. We approximate the response of this two-component system by adding a Drude term to the dielectric function of Eq. (1)

$$
\varepsilon(\omega)=\varepsilon_{X}+\frac{n_{e h} e^{2}}{\varepsilon_{0} m} \frac{1}{\omega^{2}-i \omega \Gamma_{D}}
$$

with the density of unbound pairs $n_{\mathrm{eh}}$, and a phenomenological scattering rate $\Gamma_{\mathrm{D}}$. With this correction, excellent agreement with the experiment is obtained for both functions $\Delta \sigma_{1}(\omega)$ and $\Delta \varepsilon_{1}(\omega)$ at all densities and throughout the broad $\mathrm{THz}$ frequency band (Fig. 2).

The resulting fit parameters are depicted in Figure 3. Part (a) shows a strong saturation of the total electron-hole pair density $n=n_{\mathrm{X}}+n_{\mathrm{eh}}$ as a function of the pump power, as expected due to bleaching of the near-infrared 1s heavy-hole line. According to our model, for densities below $n=5 \times 10^{10} \mathrm{~cm}^{-2}$, mainly excitons are generated. With $n$ increasing, the population share of unbound carriers becomes more prominent. Beyond a critical density $n_{\mathrm{C}}=2 \times 10^{11} \mathrm{~cm}^{-2}$, photoexcitation creates primarily unbound $e-h$ pairs. An excitonic resonance is absent and the $\mathrm{THz}$ response can be fully explained by the Drude model.

Most importantly, the renormalization of the excitonic fine structure with increasing photoexcitation is quantitatively described via the resonance frequency $E_{1 s-2 p}$ and the broadening parameter $\Gamma_{1 \mathrm{~s}-2 \mathrm{p}}$ [see Fig. 3(c) and (d), respectively]. The curves reveal a strong monotonous redshift and broadening of the $\mathrm{THz}$ resonance.

Density-dependent many-body interactions also renormalize the near-infrared interband resonance [32]. For comparison with the $\mathrm{THz}$ data, open circles in Fig. 3(c) indicate the energy shift of the near-infrared $1 \mathrm{sHH}$ resonance. Since the net blue shift is 
comprised of an elusive combination of several counteracting effects, such as band gap renormalization, phase-space filling and screening, its interpretation requires sophisticated model assumptions. In contrast, the intra-excitonic transitions observed at $\mathrm{THz}$ frequencies are unaffected by band-gap shifts and directly gauge the weakening of the binding energy.

Fig. 3(d) compares the density-dependent broadening $\Gamma_{\text {NIR }}$ of the near-infrared 1sHH absorption line with $\Gamma_{\mathrm{THz}}$. Remarkably, broadening of the $\mathrm{THz}$ response is about four times larger. We suggest that this finding results from enhanced sensitivity of the $2 p$ exciton, not visible in near-infrared spectra, to screening and scattering: its radial extent (expectation value $\left\langle\mathrm{r}_{2 \mathrm{p}}\right\rangle=58 \mathrm{~nm}$, obtained from our exciton model in the dilute limit) is six times that of $1 \mathrm{~s}$ excitons. The combination of near-infrared and $\mathrm{THz}$ probes allows for a first direct observation of this enhancement.

The experimental accessibility of the complex broadband response function imposes strict limits on the model description. The above phenomenological two component model thus reproduces the $\mathrm{THz}$ data remarkably well. Quantum many-body theories have addressed the higher-order interplay of excitons with unbound $e$-h pairs $[1,33]$. We anticipate that our studies will stimulate further developments and quantitative comparison between the $\mathrm{THz}$ response and many-body theories of $e$ - $h$ gases.

\section{Stimulated THz emission from internal exciton transitions in $\mathrm{Cu}_{2} \mathrm{O}$}

So far, we have studied absorption of $\mathrm{THz}$ photons promoting ground state excitons into higher bound energy levels. Theoretical work suggests how the underlying elementary 
processes may be effectively reversed to induce $\mathrm{THz}$ gain from inverted exciton populations $[34,35]$. Yet, stimulated emission of $\mathrm{THz}$ radiation connecting internal exciton levels had not been observed experimentally. One of the main complications comes from efficient non-radiative processes, such as scattering with phonons, excitons and free carriers, which lead to ultrafast relaxation to the lowest bound exciton level. These processes and $\mathrm{THz}$ absorption into higher bound and continuum states compete with stimulated emission and are not well understood.

Here, we show that few-cycle $\mathrm{THz}$ probe pulses provide the necessary time resolution to unravel the ultrafast dynamics of such processes. We discuss experiments that have observed the first stimulated emission of electromagnetic radiation from internal excitonic transitions [18]. The far-infrared response of the semiconductor $\mathrm{Cu}_{2} \mathrm{O}$ is monitored upon resonant generation of $3 p$ excitons. Stimulated emission from the $3 p$ to the energetically lower 2 s level manifests itself by a negative change of the absorption coefficient at a photon energy of $6.6 \mathrm{meV}$.

The semiconductor $\mathrm{Cu}_{2} \mathrm{O}$ forms a particularly well-defined laboratory for these experiments. Since excitons in $\mathrm{Cu}_{2} \mathrm{O}$ are counted among the most serious candidates for Bose Einstein condensation [8], the system has been extensively investigated by photoluminescence [8], Raman [36], mid-infrared [37], and high-resolution spectroscopy [38]. The visible absorption spectrum of our $330-\mu$ m-thick, naturally grown single-crystal is dominated by a series of sharp lines originating from the $2 p, 3 p, 4 p$, and $5 p$ excitons of the yellow series, below the pair continuum (see Fig. 4). Due to the positive parity of the valence and conduction band minima at the Brillouin zone center, even parity s and $\mathrm{d}$ 
excitons are dipole forbidden [39]. Figure 4(a) shows a detailed exciton term scheme, with the energy difference to the $3 p$ level given on the left.

An ultrafast Ti:sapphire regenerative amplifier provides 42 -fs light pulses at a center wavelength of $800 \mathrm{~nm}$ and a repetition rate of $250 \mathrm{kHz}$. These pulses simultaneously serve as the pump for an optical parametric amplifier (OPA) and drive optical rectification and electro-optic sampling in 500- $\mu \mathrm{m}$-thick ZnTe elements. Nearbandwidth-limited light pulses tunable around a wavelength of $575 \mathrm{~nm}$ (corresponding to a photon energy of $2.16 \mathrm{eV}$ ) are obtained from the OPA and a subsequent pulse shaper.

We selectively excite the $\mathrm{Cu}_{2} \mathrm{O}$ sample either resonantly at specific exciton lines or in the pair continuum and follow the subsequent transient changes in the $\mathrm{THz}$ response. A typical pump spectrum is indicated by the dashed curve in Fig. 4. Part (b) and (c) show the transmitted probe field $E_{\mathrm{THz}}(t)$ without photoexcitation (gray lines, downscaled by a factor of 500), and its transient change $\Delta E_{\mathrm{THz}}(\Delta t, t)$ induced by optical pumping at two different wavelengths. The time delay between the yellow pump and the electro-optic gating pulse is kept fixed at $\Delta t=1 \mathrm{ps}$. The response reveals critical wavelength dependence: Excitation in the continuum [Fig. 4(b)] induces electric field transients that resemble the reference pulse with a phase offset. In contrast resonant excitation of $3 p$ excitons shows a markedly different shape of the induced field. In particular, we find a striking phase shift by $\pi$ [see blue arrow in Fig. 4(c)]. We will see below that this unusual field trace is a fingerprint of stimulated $\mathrm{THz}$ emission.

It is illuminative for these experiments to discuss instead of the complex $\mathrm{THz}$ conductivity a derived quantity: the pump-induced change $\Delta \widetilde{n}(\omega)=\Delta n(\omega)+i(\mathrm{c} / 2 \omega) \Delta \alpha(\omega)$ 
of the complex refractive index. Here $\Delta n$ denotes the differential change of the real part of the refractive index and $\Delta \alpha$ the absorption change. Figure 5 displays $\Delta n$ and $\Delta \alpha$ obtained from the traces in Fig. 4. Continuum excitation [Figs. 5 (a),(b)] results in a predominantly inductive $\mathrm{THz}$ response, well explained by the Drude theory (dasheddotted lines) of a conducting $e$-h gas. In contrast, selective excitation of the $3 p$ interband resonance yields a pronounced spectral structure in both $\Delta \alpha$ and $\Delta n$. With increasing frequency, a peak of enhanced absorption occurs at $4.5 \mathrm{meV}$, followed by a narrow minimum of $\Delta \alpha$ at $6.6 \mathrm{meV}$ [Fig. 5 (d)]. Indeed, here the induced absorption becomes negative. $\Delta n$ [Fig. 5 (c)] displays a steep negative slope at a frequency of $4.5 \mathrm{meV}$, characteristic of a strong absorption line. In contrast, the dispersion of $\Delta n$ at the $\mathrm{THz}$ photon energy of $6.6 \mathrm{meV}$ has an anomalous positive gradient.

These findings consistently identify the negative absorption peak as stimulated $\mathrm{THz}$ emission via the $3 p \rightarrow 2 s$ intra-excitonic transition. This conclusion is further substantiated by the following three key observations: (i) unexcited $\mathrm{Cu}_{2} \mathrm{O}$ shows no discernible $\mathrm{THz}$ absorption in this frequency range, (ii) the minimum with $\Delta \alpha<0$ appears exclusively after resonant excitation of $3 p$ excitons, and (iii) the observed resonance energy of 6.6 $\mathrm{meV}$ is precisely identical with the $3 \mathrm{p}-2 \mathrm{~s}$ level spacing (Fig. 4).

For further analysis, we model the lineshape of the $\mathrm{THz}$ response upon $3 \mathrm{p}$ excitation. An accurate representation of the experiment is achieved with a model of three oscillators [dashed lines, Figs. 5 (c), (d)]. By comparison with the term scheme of Fig. 4 we attribute the lowest-frequency oscillator centered at $4.5 \mathrm{meV}$ to the joint effects of $3 \mathrm{p}$ $4 \mathrm{~s}$ and $3 \mathrm{p}-4 \mathrm{~d}$ transitions. The center oscillator at $6.6 \mathrm{meV}$ exhibits negative oscillator strength, in accordance with the $3 p-2 s$ stimulated emission. Finally, the component at 9.5 
$\mathrm{meV}$ phenomenologically describes all transitions to higher bound states. The relative oscillator strengths $\mathrm{f}_{1}: \mathrm{f}_{2}: \mathrm{f}_{3}=2.7:(-1): 1.1$ agree qualitatively with a hydrogen model [4.3:(1):2], thus supporting this assignment [40]. A more rigorous theory should also take into account the partial d-character of the $2 \mathrm{~s}$ exciton in $\mathrm{Cu}_{2} \mathrm{O}$, as described in Ref. [39]. With an estimated density of $3 p$ excitons of $3 \times 10^{13} \mathrm{~cm}^{-3}$ we obtain a gain cross section for the $3 p \rightarrow 2 \mathrm{~s}$ transition of $\sigma \sim 10^{-14} \mathrm{~cm}^{2}$, well in line with order-of-magnitude estimates based on hydrogen-like exciton wave functions [34].

Observation of stimulated $\mathrm{THz}$ emission critically depends on a high temporal resolution, as seen in Fig. 5(e). The induced response of electron-hole pairs is shown to exponentially decay increasingly faster as we turn from unbound states $(\tau=36 \mathrm{ps})$, via the $3 p(\tau=1.3 p s)$ to the $2 p$ exciton ( $\tau=0.8 p s)$. The relative spectral shape does not vary strongly in time (not shown). The respective relaxation times are explained by recombination and scattering of excitons into the long-lived 1s state mediated by optical phonon interactions [36] and confirm the assignment of the THz resonances.

\section{THz generation by exciton quantum beats}

Under modified excitation conditions, coherent quantum beats of $3 p$ and $2 s$ states lead to $\mathrm{THz}$ generation even without stimulation by external $\mathrm{THz}$ fields. For this set of experiments, the pump spectrum is tuned and broadened to overlap simultaneously the $3 p$ and the dipole-forbidden 2s resonances [Fig. 6(a)]. No THz probe light is incident on the sample. 
We observe emission of a coherent THz field shown in Fig. 6(b). The transient is detected electro-optically by scanning the delay $\Delta t$ between the visible pump and the gating pulse. The corresponding power spectrum in Fig. 6(c) peaks at $6.6 \mathrm{meV}$ and exhibits a width of $1.7 \mathrm{meV}$ (FWHM). This lineshape coincides with the values deduced from the 3p-2s transition in Fig. 5(d), corroborating that the $\mathrm{THz}$ transient originates from the same internal exciton transition. The absolute size of these emitted fields is comparable to the transients $\Delta E_{\mathrm{THz}}$ in Fig. 4 stimulated by a far-infrared seed pulse. This fact implies that the generation mechanism cannot be explained by a spontaneous version of the gain process discussed above. Moreover, we find that the $\mathrm{THz}$ polarization is parallel to the linear polarization of the pump light, whereas the polarization of the stimulated change $\Delta E_{\mathrm{THz}}$, described further above, is determined by the probe pulses.

We interpret this $\mathrm{THz}$ emission as resulting from quantum beats between coherent exciton polarizations. Visible light induces $3 p$ exciton polarizations, while the low-energy wing of the pump spectrum couples the 2 s level to the ground state, e.g. via a weak quadrupole or surface field assisted dipole transition [34]. As $3 p$ and $2 s$ states possess opposite parity, quantum beats between them act as a radiating dipole. The $\mathrm{THz}$ generation may be expressed as an effective difference frequency process (see insert of Fig. 6), described by the nonlinear susceptibility

$$
\chi_{\text {eff }}^{(2)}(\omega) \propto \frac{M_{3 p} M_{2 s} M_{3 p-2 s}}{\left(\omega-\omega_{3 p}+i \Gamma_{3 p}\right)\left(\omega_{T H z}-\omega_{3 p-2 s}+i \Gamma_{3 p-2 s}\right)}
$$

where $M_{3 \mathrm{p}}$ and $M_{2 \mathrm{~s}}$ are the visible interband matrix elements, $M_{3 \mathrm{p}-2 \mathrm{~s}}$ is the THz dipole matrix element. $\omega$ and $\omega_{\mathrm{THz}}$ are the frequencies of visible pump and emitted THz fields, 
and $\omega_{3 p}$ and $\omega_{3 p-2 s}$ are visible $3 p$ and $\mathrm{THz} 3 p-2 s$ resonance energies with corresponding dephasing constants $\Gamma_{\mathrm{i}}$. Equation (3) follows from a simplified treatment of stimulated polariton scattering [41].

In our experiment, one of the denominator terms is chosen resonant by the pump photon energy $\left(\omega=\omega_{3 \mathrm{p}}\right)$, and THz emission is observed whenever $\omega_{\mathrm{THz}}=\omega_{3 \mathrm{p}-2 \mathrm{~s}}$. For this $\mathrm{THz}$ frequency both terms in the denominator are resonant, overcoming the weakness of the coupling between $2 \mathrm{~s}$ excitons and the ground state. Off-resonant pumping reduces the conversion efficiency strongly as demonstrated below. The emitted $\mathrm{THz}$ intensity scales with the square of the pump power (not shown) characteristic of a $\chi^{(2)}$ effect. From the pulse intensities and effective interaction length we estimate a resonant nonlinearity of $\chi_{\text {eff }}^{(2)} \geq 10 \mathrm{pm} / \mathrm{V}$. This value exceeds the high background nonlinearity of noncentrosymmetric materials such as $\mathrm{ZnTe}$, which is widely used for $\mathrm{THz}$ generation [28].

The above principle of $\mathrm{THz}$ emission may be extended to map out the internal fine structure of excitons in a systematic way. By detuning the pump spectra from the one depicted in Fig. 6(a), one may induce a coherent superposition of various alternative pairs of excitonic quantum states. This idea is implemented in Fig. 7. The power spectra of the emitted THz radiation exhibits a resonant enhancement of $\chi^{(2)}$ whenever a pair of exciton states of opposite parity is excited: The THz spectrum of Fig. 6 is reproduced for a pump spectrum centered at $2.158 \mathrm{eV}$ [see also Fig. 6(a)] due to coherent quantum beats of $2 \mathrm{~s}$ and $3 p$ excitons. At lower pump photon energies, THz emission becomes inefficient and spectrally less selective as expected from Eq. (3), since the resonance denominator is no longer singular. On the other hand, if the pump photons have sufficient energy to drive alternative pairs of excitonic states, such as $(4 \mathrm{p}, 3 \mathrm{~s})$ or $(4 \mathrm{p}, 3 \mathrm{~d}), \chi^{(2)}$ is resonantly 
enhanced at the corresponding THz photon energies. In fact, the spectra of Fig. 7 clearly reflect this situation for intra-excitonic transitions $3 p-2 s, 4 p-3 s$, and $4 p-3 d$. Interestingly, $\mathrm{THz}$ generation is most efficient for the $3 \mathrm{p}-2 \mathrm{~s}$ quantum beat. This observation may be explained by the relative size of the matrix elements $M_{\mathrm{i}}$ which are expected to decrease rapidly with increasing principal quantum number [2]. Our novel spectroscopy scheme may become a helpful tool to systematically map out the internal fine structure of excitons also in other semiconductor systems.

\section{Conclusion}

We have used broadband $\mathrm{THz}$ spectroscopy to study the ultrafast dynamics, spectral positions, widths and oscillator strengths of intra-excitonic transitions. In a twodimensional exciton gas in GaAs quantum wells, the density-dependent renormalization of the excitonic fine structure was directly recorded by probing the $\mathrm{THz}$ absorption from the $1 \mathrm{~s}-2 \mathrm{p}$ transition. Furthermore, we have demonstrated the inverse quantum process, stimulated $\mathrm{THz}$ emission between the $3 \mathrm{p}$ and $2 \mathrm{~s}$ exciton states in $\mathrm{Cu}_{2} \mathrm{O}$ with a significantly large cross section. This underpins the analogy between excitons and atoms. A novel intra-excitonic quantum beat spectroscopy, finally, allows us to map out an extended set of transitions between several excited states. While the experiments discussed here focus on far-infrared exciton lines, latest advances in ultra-broadband nonlinear optics pave the way for $\mathrm{THz}$ studies of more strongly bound systems with high thermal stability.

The experiments yield novel insight into the complex phase diagram of excitons and provide access to key quantum optical processes. These are key ingredients for future 
$\mathrm{THz}$ quantum optical manipulations of excitons outside the optically accessible range. Hence, THz opto-electronics may become a helpful tool in the ongoing quest for excitonic Bose Einstein condensation.

\section{Acknowledgements.}

We thank Y. R. Shen, C. Kübler, A. Leitenstorfer, N. C. Nielsen, and T. Zentgraf for helpful discussions and continuous support. J. Reno is acknowledged for providing quantum-well samples. This work was supported by the Director, Office of Science, Office of Basic Energy Science of the US Department of Energy under Contract No. DEAC02-05CH11231. R.H. is grateful for support from the Alexander von Humboldt Foundation and the German Research Foundation through the Emmy Noether Program. 


\section{References}

1. R. Zimmermann, Many-Particle Theory of Highly Excited Semiconductors, Teubner-Texte zur Physik, Vol. 18 (BSB Teubner, Berlin, 1987).

2. H. Haug and S. W. Koch, Quantum Theory of the Optical and Electronic Properties of Semiconductors (World Scientific, Singapore, 2004).

3. I. Perakis, Nature 417, 33 (2002) and references therein.

4. N.F. Mott, Metal Insulator Transitions (Taylor and Francis, London, 1990).

5. L. Kappei, J. Szczytko, F. Morier-Genoud, B. Deveaud, Phys. Rev. Lett. 94, 147403 (2005).

6. L.V. Butov, C.W. Lai, A.L. Ivanov, A.C. Gossard, D.S. Chemla, Nature 417, 47 (2002); L.S. Levitov, B.D. Simons, L.V. Butov, Phys. Rev. Lett. 94, 176404 (2005).

7. D. Snoke, S. Denev, Y. Liu, L. Pfeiffer, K. West, Nature 418, 754 (2002).

8. D. W. Snoke, J.P. Wolfe, A. Mysyrowicz, Phys. Rev. Lett. 64, 2543 (1990); K.E. O'Hara, J.P. Wolfe, Phys. Rev. B 62, 12909 (2000).

9. M. Kira, W. Hoyer, T. Stroucken, S.W. Koch, Phys. Rev. Lett. 87, 176401 (2001).

10. J. Szczytko, et al., Phys. Rev. Lett. 93, 137401 (2004); Phys. Rev. B 71, 195313 (2005).

11. S. Chatterjee et al., Phys. Rev. Lett. 92, 067402 (2004).

12. R.H.M. Groeneveld, D. Grischkowsky, J. Opt. Soc. Am. B 11, 2502-2507 (1994).

13. J. Cerne, J. Kono, M.S. Sherwin, M. Sundaram, A.C. Gossard, G.E.W. Bauer, Phys. Rev. Lett. 77, 1131 (1996).

14. R. Huber, F. Tauser, A. Brodschelm, M. Bichler, G. Abstreiter and A. Leitenstorfer, Nature 414, 286 (2001).

15. R.A. Kaindl, M.A. Carnahan, D. Hägele, R. Lövenich, D.S. Chemla, Nature 423, 734 (2003).

16. R. Huber, et al., Phys. Rev. Lett. 94, 027401 (2005).

17. R. Huber, R. A. Kaindl, B. A. Schmid, and D. S. Chemla, Phys. Rev. B. 72, 161314(R) (2005).

18. R. Huber, B. A. Schmid, Y. R. Shen, D. S. Chemla, and R. A. Kaindl, Phys. Rev. Lett. 96, 017402 (2006).

19. M. Born and E. Wolf, Principles of Optics (Pergamon Press, New York, 1980).

20. For a review see, e.g., B. Ferguson and X.-C. Zhang, Nat. Mater. 1, 26 (2002) and references therein.

21. R. A. Kaindl, et al., Opt. Lett. 23, 861-863 (1998); R. A. Kaindl, F. Eickemeyer, M Woerner, and T. Elsaesser, Appl. Phys. Lett. 75, 1060 (1999). 
22. R. Huber, A. Brodschelm, F. Tauser, and A. Leitenstorfer, Appl. Phys. Lett. 76, 3191 (2000)).

23. C. Kübler, R. Huber, S. Tübel and A. Leitenstorfer, Appl. Phys. Lett. 85, 3360 (2004).

24. T. Zentgraf, R. Huber, N. C. Nielsen, D. S. Chemla, and R. Kaindl, Opt. Expr. 15, 5775 (2007).

25. F. Eickemeyer, R. A. Kaindl, M. Woerner, T. Elsaesser, and A.M. Weiner, Opt. Lett. 25, 1472 (2000).

26. T. Bartel, P. Gaal, K. Reimann, M. Woerner, and T. Elsaesser, Opt. Lett. 30, 2805 (2005).

27. Ch. Fattinger, and D. Grischkowsky, Appl. Phys. Lett. 54, 490 (1989).

28. Q. Wu and X.-C. Zhang, Appl. Phys. Lett. 68, 1604 (1996).

29. M. C. Beard, G. M. Turner, and C. A. Schmuttenmaer, Phys. Rev. B 62, 15764 (2000).

30. D. S. Chemla and J. Shah, Nature 411, 549 (2001).

31. R. A. Kaindl, D. Hägele, M. A. Carnahan, R. Lövenich, and D. S. Chemla (unpublished).

32. see e.g. G. W. Fehrenbach, W. Schäfer, J. Treusch, and R. G. Ulbrich, Phys. Rev. Lett. 49, 1281 (1982); N Peyghambarian, et al., Phys. Rev. Lett. 53, 2433 (1984); W. H. Knox, et al., Phys. Rev. Lett. 56, 1191 (1986); G. Manzke, et al., Phys. Rev. Lett. 80, 4943 (1998).

33. M. Kira and S. W. Koch, Many-body correlations and excitonic effects in semiconductor spectroscopy, in G. Eden and H. Jelnikova, Progress in Quantum Electronics, Vol. 30 (Elsevier, Amsterdam, 2006).

34. S. Nikitine, J. Phys. Chem. Solids 45, 949 (1984).

35. M. Kira and S. W. Koch, Phys. Rev. Lett. 93, 076402 (2004).

36. P. Y. Yu and Y. R. Shen, Phys. Rev. B 17, 4017 (1978).

37. M. Kubouchi, K. Yoshioka, R. Shimano, A. Mysyrowicz, M. Kuwata-Gonokami, Phys. Rev. Lett. 94, 016403 (2005).

38. D. Fröhlich, J. Brandt, C. Sandfort, M. Bayer, and H. Stolz, phys. stat. sol. (b) 64, 2543 (1994); G. Dasbach, et al., Phys. Rev. Lett. 91, 107401 (2003).

39. C. Uihlein et al., Phys. Rev. B 14, 1772 (1976).

40. H. A. Bethe and E. E. Salpeter, Quantum Mechanics of One- and Two-Electron Atoms (Academic Press, New York, 1957).

41. Y. R. Shen, The Principles of Nonlinear Optics (Wiley, New York, 2002). 


\section{Figure Captions}

FIG 1 (a)-(c) Broadband phase-locked THz transients generated via optical rectification and detected by electro-optic sampling in nonlinear optical crystals as follows: (a) emitter and detector: (110)-GaP $(\mathrm{d}=200 \mu \mathrm{m}),(\mathrm{b})$ emitter: GaSe $(\mathrm{d}=100 \mu \mathrm{m})$, detector: (110)$\operatorname{ZnTe}(\mathrm{d}=15 \mu \mathrm{m}),(\mathrm{c})$ emitter and detector: GaSe $(\mathrm{d}=30 \mu \mathrm{m})$, data from Ref. [23], (d) corresponding amplitude spectra (i)-(iii) continuously covering the far- and mid-infrared regime. Spectrum (iv) corresponds to a phase-locked pulse generated by phase-matched optical rectification of a 7-fs near-infrared pulse in a $\mathrm{LiIO}_{3}$ element [24].

FIG 2 (a) Near-infrared absorption spectrum of the quantum well sample at $T_{\mathrm{L}}=6 \mathrm{~K}$ (solid curve). The pump spectrum (dotted curve) is resonant with the 1s heavy-hole interband line. (b) Photoinduced conductivity $\Delta \sigma_{1}(\omega)$ and (c) dielectric function change $\Delta \varepsilon_{1}(\omega)$ for various $e-h$ pair densities $n$ in units of $n_{0}=10^{10} \mathrm{~cm}^{-2}$. The data are taken at $\Delta t$ $=2.5$ ps. Solid curves: two-fluid model of Eq. (2); dashed curves at $n=20 \times n_{0}$ : quasi-2D exciton model of Eq. (1). Curves for different densities are equally scaled and vertically shifted.

FIG 3 Fit parameters of the two-component model to the THz response of Fig. 2. (a) Total electron-hole pair density $n$ as function of excitation power. (b) Free carrier density $n_{\text {eh }}$ as a function of $n$. (c) Intra-excitonic level spacing $E_{1 s-2 p}$ (filled squares) measured via $\mathrm{THz}$ spectroscopy, and density-induced energy shift $\Delta \mathrm{E}_{\mathrm{NIR}}$ (open circles) of the interband 
resonance. (d) Filled squares: Full width at half maximum $\Gamma_{\mathrm{X}}$ of the $\mathrm{THz}$ resonance; open circles: FWHM $\Gamma_{\text {NIR }}$ of the near-infrared absorption line. The solid curves are guides to the eye.

FIG 4 (a) Optical absorption spectrum of the $\mathrm{Cu}_{2} \mathrm{O}$ crystal studied in this work $\left(T_{\mathrm{L}}=6\right.$ K). Inset: term scheme of yellow exciton series [39]. Dashed curve: typical pump spectrum for resonant $3 p$ excitation (thick upward arrow in inset). Internal exciton transitions from $\mathrm{p}$ to $\mathrm{s}$ and $\mathrm{d}$ states are dipole allowed (solid arrow). (b) and (c): Pump induced changes $\Delta E_{\mathrm{THz}}$ (black and blue) at $\Delta t=1 \mathrm{ps}$ and reference $\mathrm{THz}$ fields $E_{\mathrm{THz}}$ (gray curves, downscaled by 500). Pump spectra are centered (b) in the continuum at $2.206 \mathrm{eV}$ and (c) resonant to the $3 p$ line. Arrow: phase slip by $\pi$.

FIG $5 \mathrm{THz}$ response at $\Delta t=1 \mathrm{ps}$ after excitation of unbound electron-hole pairs and $3 \mathrm{p}$ excitons. (a), (c) Changes of refractive index $\Delta n$ and (b), (d) absorption coefficient $\Delta \alpha$ (solid lines). Dash-dotted curves: Drude response. Dashed lines: model of three oscillators with energies $E_{1}=4.5 \mathrm{meV}, E_{2}=6.6 \mathrm{meV}$, and $E_{3}=9.5 \mathrm{meV}$, and relative oscillator strengths as given in the text. The vertical arrow marks the negative absorption change occurring around $6.6 \mathrm{meV}$. (e) Ultrafast pump-probe dynamics of the induced change of the $\mathrm{THz}$ field at various pump energies.

FIG $6 \mathrm{THz}$ generation in $\mathrm{Cu}_{2} \mathrm{O}$ : (a) Pump spectrum (solid black line), (b) real-time trace, and (c) normalized power spectrum of the THz transient (FWHM: $1.7 \mathrm{meV}$ ). The spectral 
width of the pump pulse is set to $12 \mathrm{meV}$ (FWHM). Inset in (c): Term scheme of the underlying quantum process.

FIG 7 Two-dimensional THz emission spectroscopy of intra-excitonic lines in $\mathrm{Cu}_{2} \mathrm{O}$. (a) $\mathrm{THz}$ spectra for a series of different pump wavelengths. All spectra are equally scaled and vertically offset to align with the center photon energy of the respective pump on the right hand scale of (b). (b) reproduces the visible absorption spectrum for comparison. The width of each pump spectrum is kept at $12 \mathrm{meV}$ (FWHM). Vertical lines in (a) mark the energy position of prominent intra-excitonic transitions. 
FIGURE 1
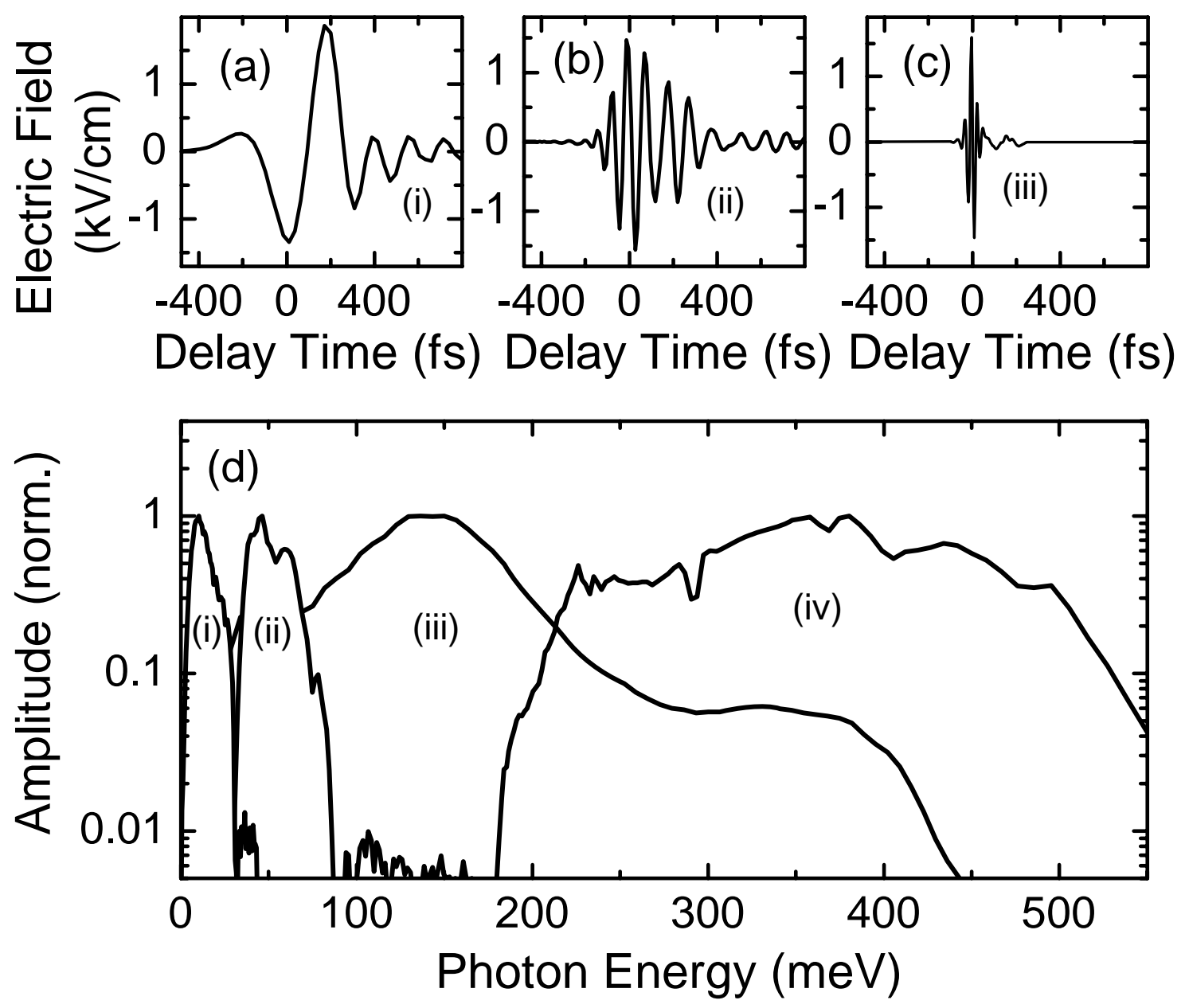
FIGURE 2

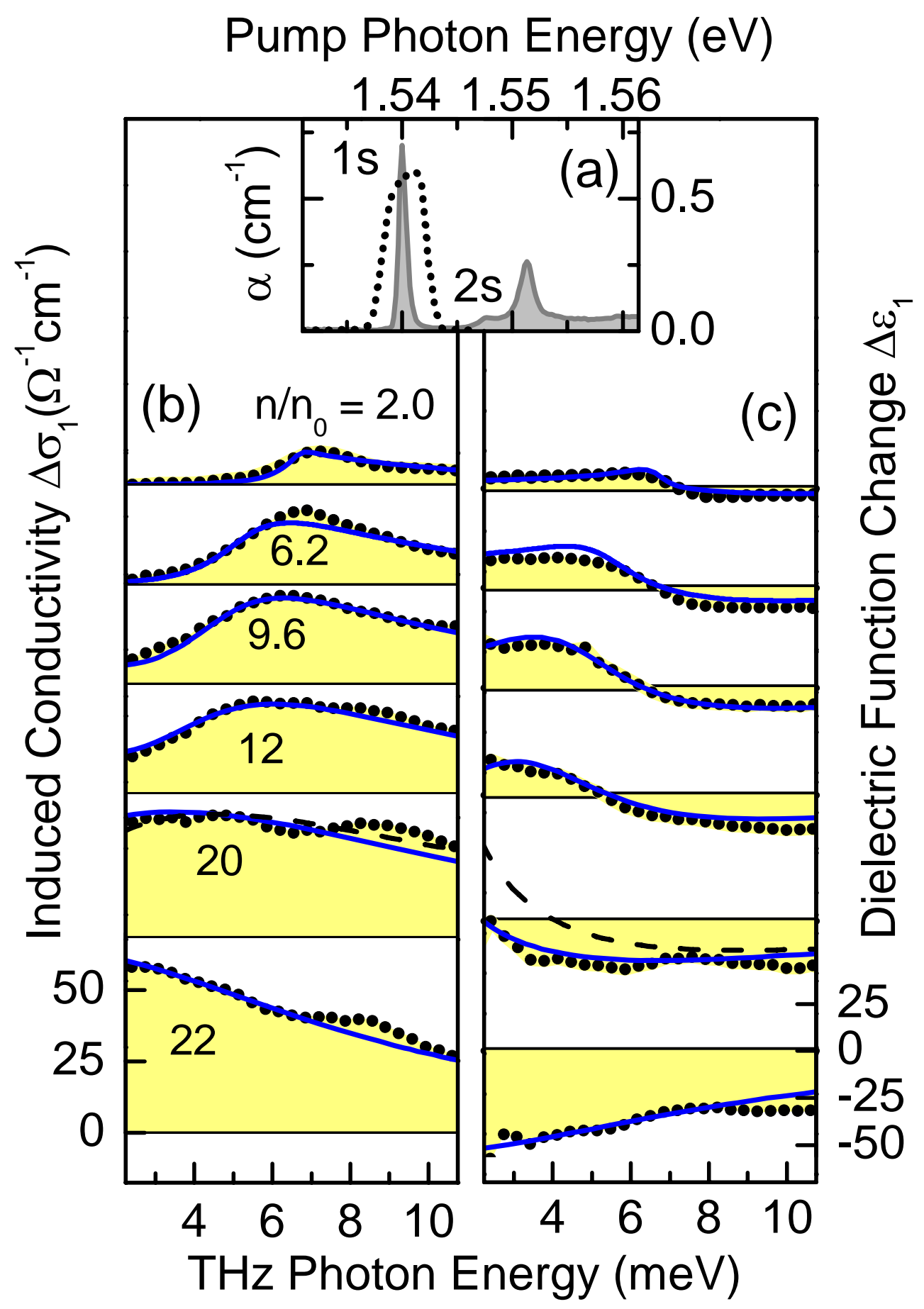


FIGURE 3
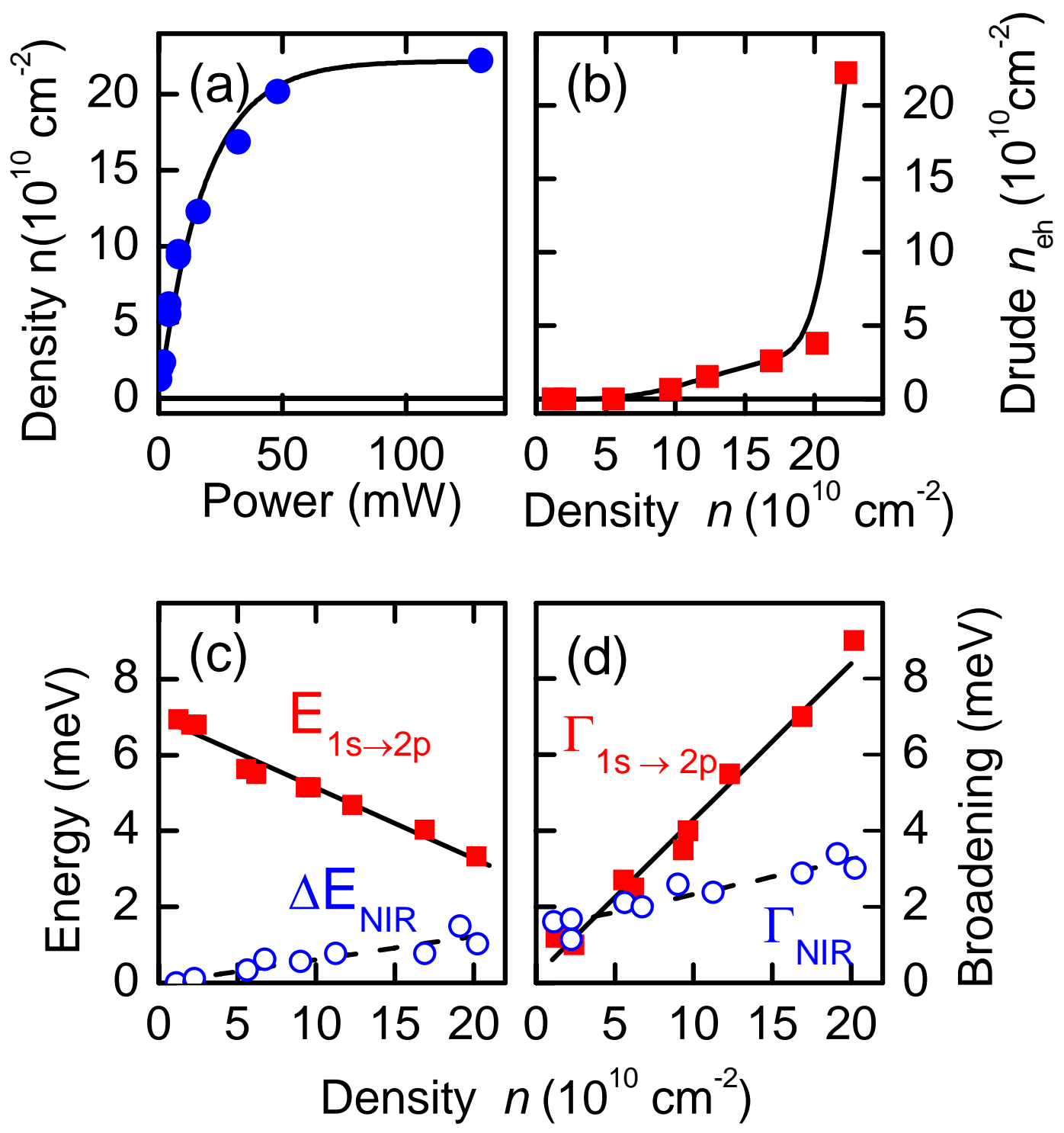
FIGURE 4
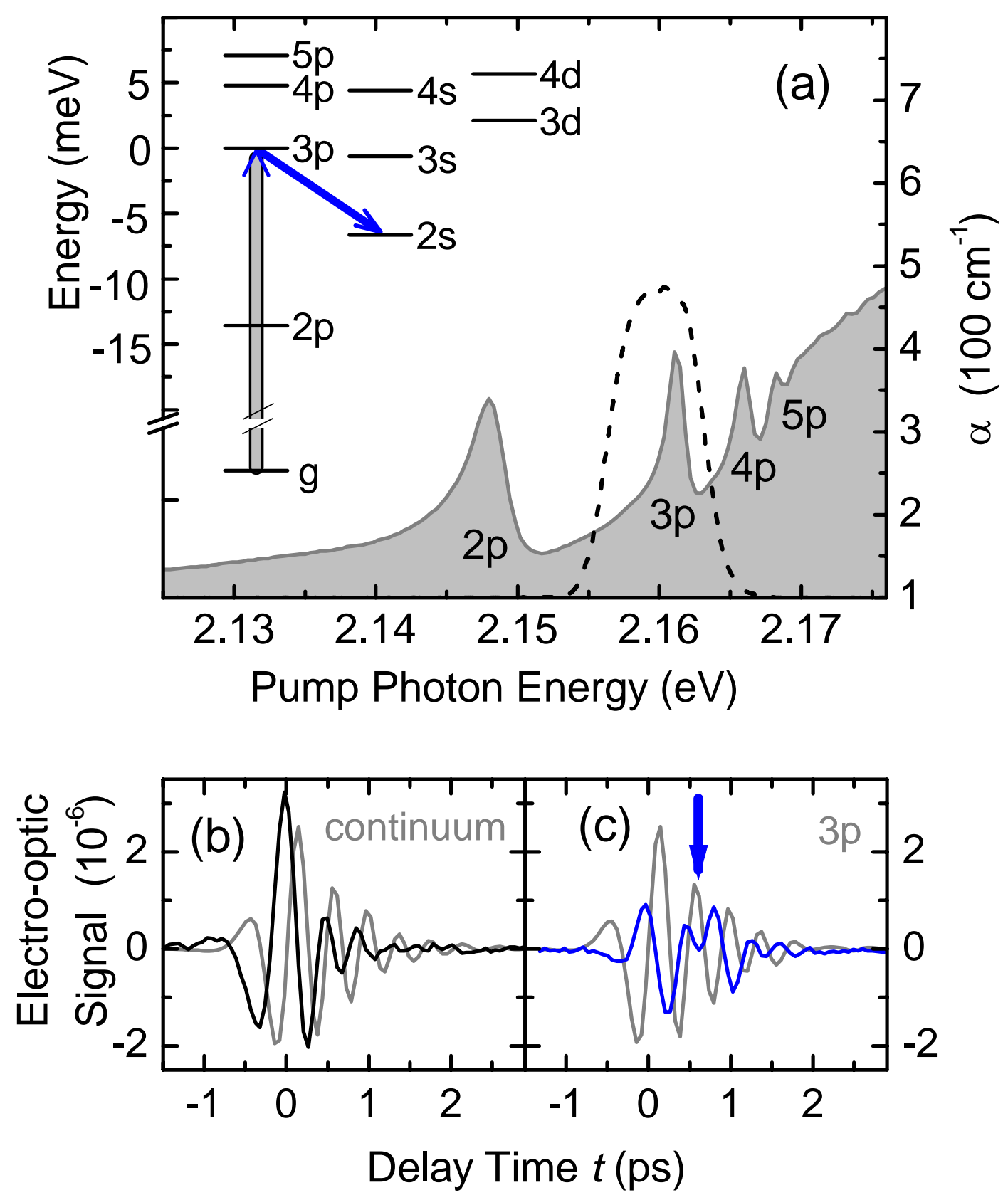
FIGURE 5
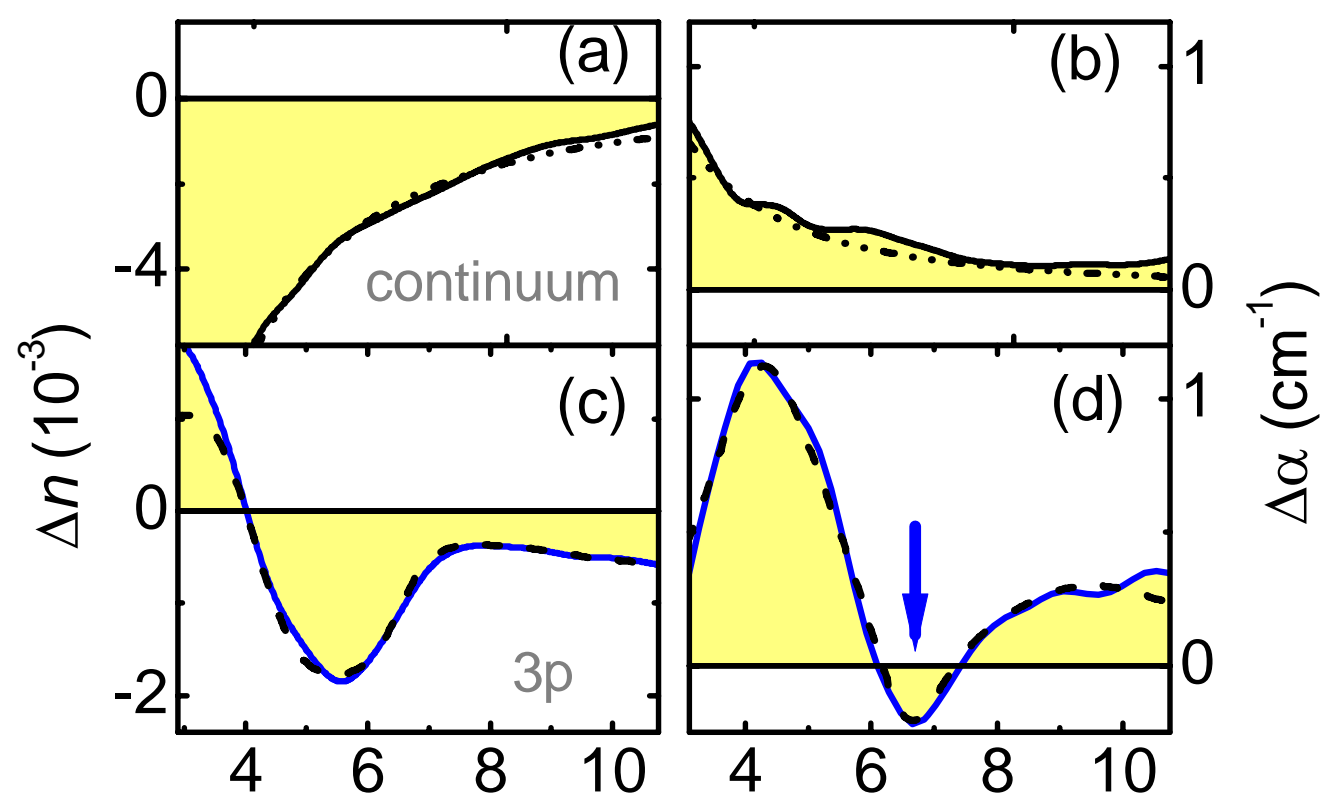

$\mathrm{THz}$ Photon Energy (meV)

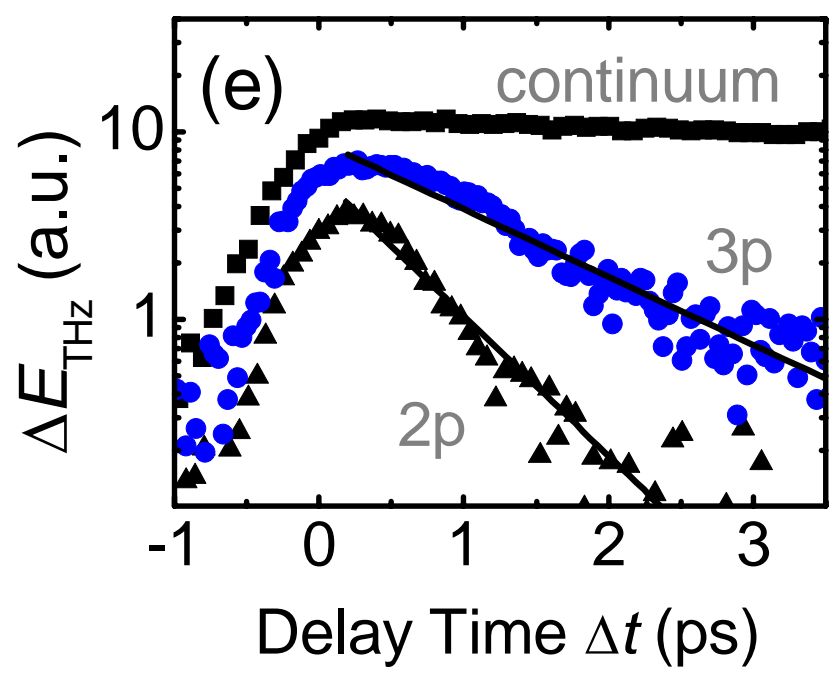


FIGURE 6

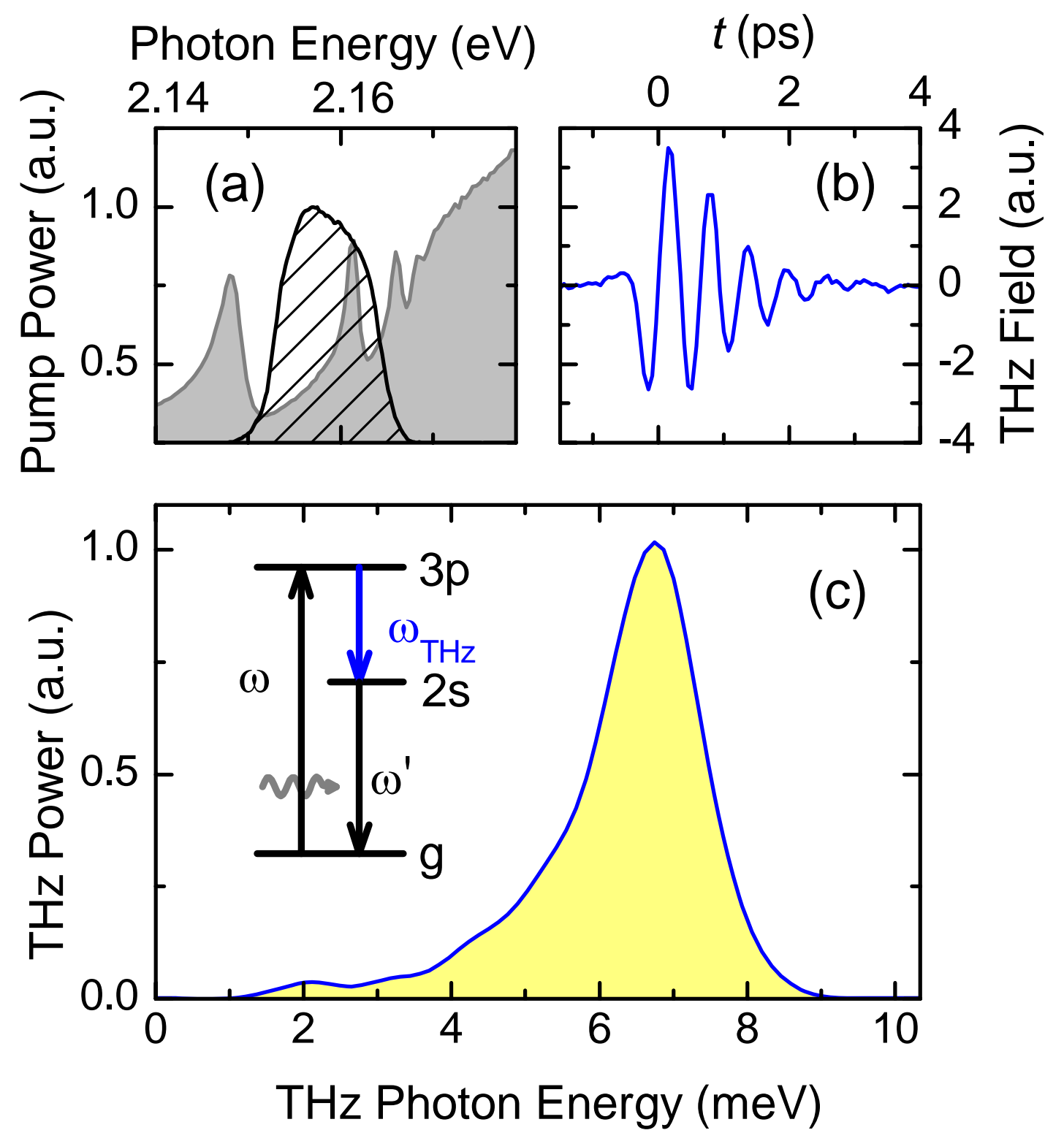


FIGURE 7
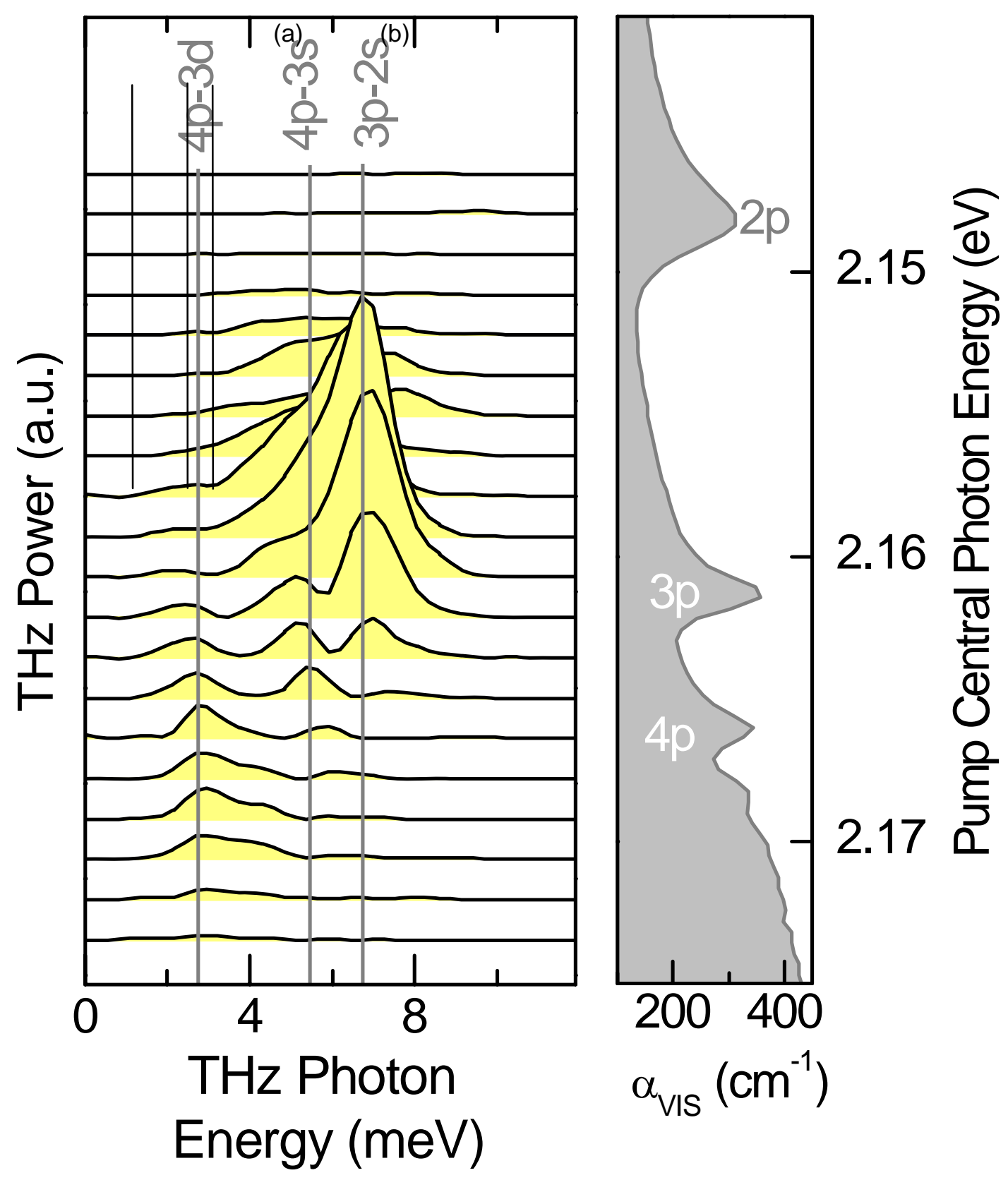


\section{DISCLAIMER}

This document was prepared as an account of work sponsored by the United States Government. While this document is believed to contain correct information, neither the United States Government nor any agency thereof, nor The Regents of the University of California, nor any of their employees, makes any warranty, express or implied, or assumes any legal responsibility for the accuracy, completeness, or usefulness of any information, apparatus, product, or process disclosed, or represents that its use would not infringe privately owned rights. Reference herein to any specific commercial product, process, or service by its trade name, trademark, manufacturer, or otherwise, does not necessarily constitute or imply its endorsement, recommendation, or favoring by the United States Government or any agency thereof, or The Regents of the University of California. The views and opinions of authors expressed herein do not necessarily state or reflect those of the United States Government or any agency thereof or The Regents of the University of California. 